\title{
A novel technique for placing titanium mesh with porous polyethylene via the endoscopic transnasal approach into the orbit for medial orbital wall fractures
}

\author{
Seong Hwan Bae ${ }^{1,2}$, Dae Kyun Jeong ${ }^{1}$, Ju Young Go ${ }^{3}$, Heeseung Park ${ }^{4}$, Joo Hyoung Kim¹, \\ Jae Woo Lee ${ }^{1}$, Taewoo Kang ${ }^{4}$ \\ ${ }^{1}$ Department of Plastic and Reconstructive Surgery, Pusan National University School of Medicine, Busan; ${ }^{2}$ Biomedical Research Institute, \\ Pusan National University Hospital, Busan; ${ }^{3}$ TAE Plastic Surgery Clinic, Busan; ${ }^{4}$ Busan Cancer Center, Pusan National University Hospital, \\ Busan, Korea
}

Background The endoscopic transnasal approach is widely used for reconstructing the medial orbital wall by filling it with a silicone sheet or Merocel, but this technique has the disadvantage of retaining the packing for a long time. To overcome this drawback, a method of positioning an absorbable plate in the orbit has been introduced, but there is a risk of defect recurrence after the plate is absorbed. Here, the authors report the results of a novel surgical technique of placing a nonabsorbable titanium mesh with porous polyethylene into the orbit through the endoscopic transnasal approach.

Methods Fourteen patients underwent surgery using the endoscopic transnasal approach. Preoperative computed tomography (CT) was used to calculate the size of the bone defect due to the fracture, and the titanium mesh was designed to be shorter than the anteroposterior length of the defect and longer than its height. The titanium mesh was inserted into the orbit under an endoscopic view. The authors then confirmed that the titanium mesh supported the orbital contents by pressing the eyeball and finished the operation. Immediately after surgery, CT results were evaluated.

Results Postoperative CT scans confirmed that the titanium mesh was well-inserted and in the correct position. All patients were discharged without any complications.

Conclusions We obtained satisfactory results by inserting a titanium mesh with porous polyethylene into the orbit via the transnasal approach endoscopically.

Keywords Wound and injuries / Endoscopy / Orbit / General surgery / Titanium
Correspondence: Taewoo Kang Busan Cancer Center, Pusan National University Hospital, 179 Gudeok-ro, Seo-gu, Busan 49241, Korea Tel: +82-51-240-7281 Fax: $+82-51-240-7746$ E-mail: taewoo.kang@pusan.ac.kr

This work was supported by a clinical research grant from Pusan National University Hospital in 2016 (a personal grant to SHB in 2016).

This article was presented at the 8th Research and Reconstruction (R\&R) forum on April 19-20, 2018, in Jeonju, Korea.

\section{INTRODUCTION}

Blowout fractures account for $13.3 \%$ of all facial fractures, and medial orbital wall fractures alone account for $0 \%$ to $55 \%$ of cases [1-3]. Although the incidence of medial orbital wall fractures is not very low, there is currently less consensus regarding the

Copyright $($ C 2019 The Korean Society of Plastic and Reconstructive Surgeons

This is an Open Access article distributed under the terms of the Creative Commons Attribution Non-Commercial License (http://creativecommons.org/

licenses/by-nc/4.0/) which permits unrestricted non-commercial use, distribution, and reproduction in any medium, provided the original work is properly cited.

www.e-aps.org 
treatment of medial orbital wall fractures than there is for orbital floor fractures [4]. A medial orbital wall fracture that is not treated correctly can cause late enophthalmos and facial asymmetry [5]. Therefore, more research is needed on the optimal treatment of this condition.

Various approaches have been used for the surgical treatment of medial orbital wall fractures. Some of these approaches have disadvantages, such as difficulty in securing sufficient vision for the operation and scarring in the facial area. The endoscopic transnasal approach introduced by Yamaguchi et al. [6] in 1991 is widely used to overcome these shortcomings [7-11].

The endoscopic transnasal approach is often used to fill the nasal cavity with a silicone sheet and Merocel to reconstruct the medial orbital wall. However, this technique has several problems; for instance, the patient must endure considerable discomfort and the risk of surgical site infection increases because the filling materials must be maintained for a long time. To overcome these drawbacks, a method of positioning an absorbable plate in the orbit through the endoscopic transnasal approach has been introduced [12]. However, it is difficult to identify absorbable plates because they are radiolucent, and they also may induce a foreign body reaction [13]. Furthermore, although this is somewhat controversial, it is likely that late enophthalmos can occur after absorption of the plate [14-16].

To overcome these disadvantages, the authors devised a new surgical technique to place a nonabsorbable plate of titanium mesh with porous polyethylene into the orbit endoscopically via the transnasal approach. Here, we report the results of applying this surgical technique in a series of cases.

\section{METHODS}

\section{Study patients}

From July 2017 to July 2018, 14 patients who were diagnosed with pure medial orbital wall fractures were operated on using the endoscopic transnasal approach. The causes of the fractures were falls in five patients, assaults in three patients, traffic accidents in five patients, and exercise injury in one patient. There were nine males and five females. Three patients complained of diplopia at the time of admission, and six patients complained of gaze limitation. All patients underwent surgery within 2 weeks of the injury (Table 1).

\section{Surgical procedure}

All patients underwent orbital wall reconstruction surgery using the endoscopic transnasal approach under general anesthesia. First, cottonoids soaked with diluted epinephrine $(1: 100,000)$ were filled in the middle meatus using a $0^{\circ} 4$-mm-diameter en-

\begin{tabular}{|c|c|c|c|c|c|}
\hline Patient & Sex & $\begin{array}{l}\text { Age } \\
\text { (yr) }\end{array}$ & Cause & $\begin{array}{c}\text { Defect } \\
\text { size }\left(\mathrm{cm}^{2}\right)\end{array}$ & Problem \\
\hline 1 & Female & 35 & Traffic accident & 1.7 & Gaze limitation \\
\hline 2 & Female & 70 & Traffic accident & 3.4 & None \\
\hline 3 & Male & 39 & Assault & 2.5 & None \\
\hline 4 & Male & 29 & Traffic accident & 2.3 & None \\
\hline 5 & Female & 59 & Fall & 3.2 & Diplopia, gaze limitation \\
\hline 6 & Male & 17 & Fall & 4.2 & None \\
\hline 7 & Male & 27 & Assault & 3.9 & None \\
\hline 8 & Male & 52 & Traffic accident & 2.3 & Gaze limitation \\
\hline 9 & Male & 67 & Assault & 3.4 & None \\
\hline 10 & Male & 43 & Exercise & 2.7 & Diplopia, gaze limitation \\
\hline 11 & Male & 56 & Traffic accident & 4.8 & None \\
\hline 12 & Female & 37 & Fall & 4.0 & Diplopia, gaze limitation \\
\hline 13 & Female & 58 & Fall & 2.2 & None \\
\hline 14 & Male & 56 & Fall & 2.9 & Gaze limitation \\
\hline
\end{tabular}

doscope (Tricam NTSC; Storz, Tuttlingen, Germany) to induce atrophy and vasoconstriction of the nasal mucosa. A solution of 1:100,000 epinephrine and 2\% lidocaine was injected into the anterior border of the middle turbinate, around the uncinate process, and into the adjacent septum and lateral wall of the middle turbinate. When needed for a sufficient visual field, the anterior part of the middle turbinate was partially resected. An incision was made in the uncinate process using a sickle knife, and the ethmoidal sinus was opened. After identifying the site of the medial orbital wall fracture, the herniated orbital contents were pushed into the orbit. Preoperative computed tomography (CT) images were used to calculate the size of the bone defect due to fracture. The size of the bone defect was calculated by multiplying the width by the height of the defect. The titanium mesh with porous polyethylene (Medpor Titan Barrier sheet; Stryker, Kalamazoo, MI, USA) was trimmed to be shorter than the anteroposterior length of the defect and longer than its height (Fig. 1). A $30^{\circ}$ endoscope was used to insert a titanium mesh into the bone defect. We placed the implant so that it could be fixed to the uninjured border of the defect (Fig. 2). After pressing the eyeball, we confirmed that the titanium mesh supported the orbital contents. Immediately after the operation, the forced duction test was performed, and the operation was terminated.

\section{Radiologic examinations}

Before and after surgery, CT was performed using the same protocol (120 kV, $280 \mathrm{mAs}, 1.0-\mathrm{mm}$ section thickness). Preoperative CT scans were taken within 1 week from the date of injury, and postoperative $\mathrm{CT}$ was performed immediately after surgery (Figs. 3-5). 


\section{Fig. 1. Titanium mesh with porous polyethylene}

A titanium mesh with porous polyethylene was inserted into the bone defect through the endoscopic transnasal approach and positioned in the orbit. The titanium mesh was trimmed to be shorter than the anteroposterior length of the defect and longer than its height.

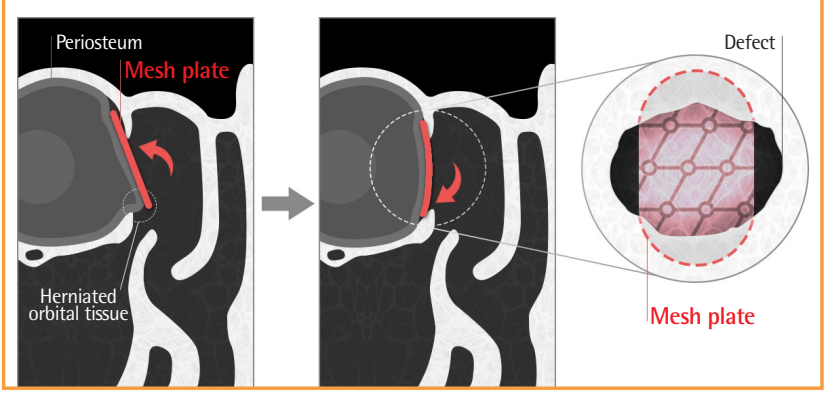

\section{Fig. 2. Intraoperative $30^{\circ}$ endoscopic view}

A case of medial blowout fracture. Medial intraoperative $30^{\circ}$ endoscopic view (A) before insertion of the titanium mesh after uncinectomy (B) after insertion of the implant. After pressing the eyeball, the titanium mesh was confirmed to support the orbital contents.
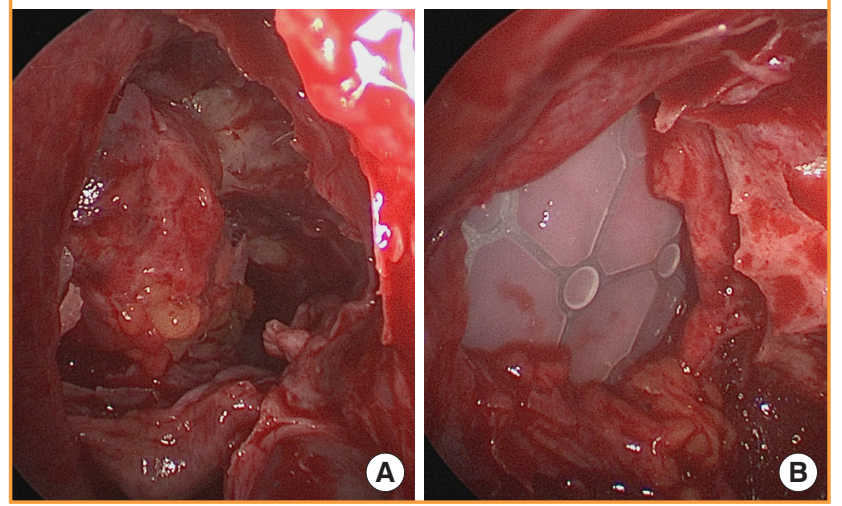

\section{Fig. 3. Axial computed tomography image}

A computed tomography image of a medial blowout fracture. (A) Preoperative. (B) Postoperative.
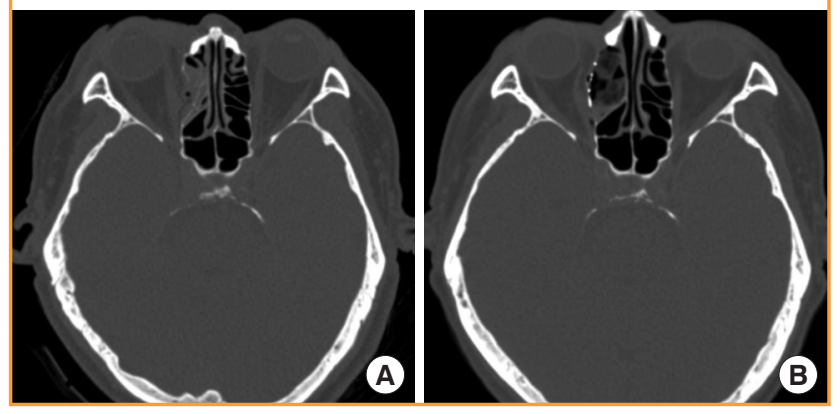

\section{Follow-up of patients}

All patients underwent follow-up at 2 weeks after surgery. The authors assessed patients for the presence of symptoms such as diplopia and gaze limitation.
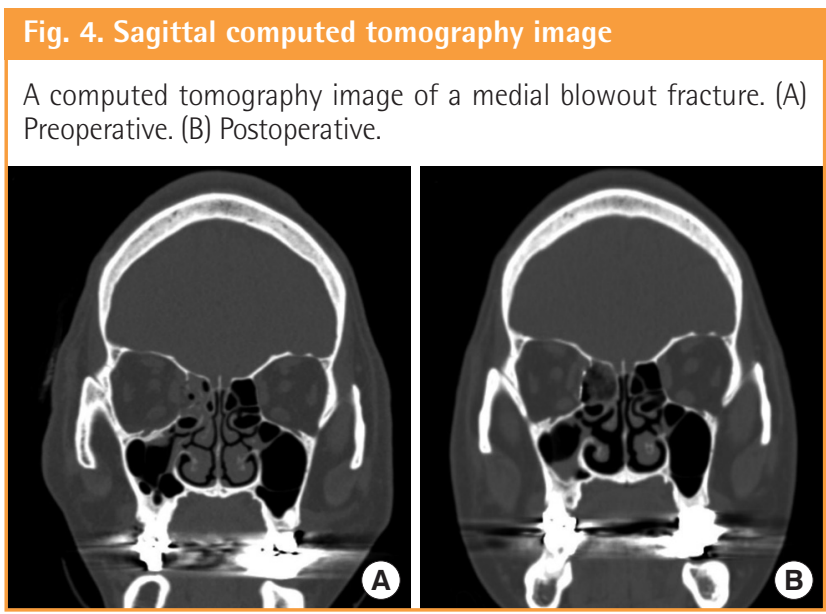

\section{Fig. 5. Three-dimensional computed tomography image}

A computed tomography image of a medial blowout fracture. (A) Preoperative. (B) Postoperative.
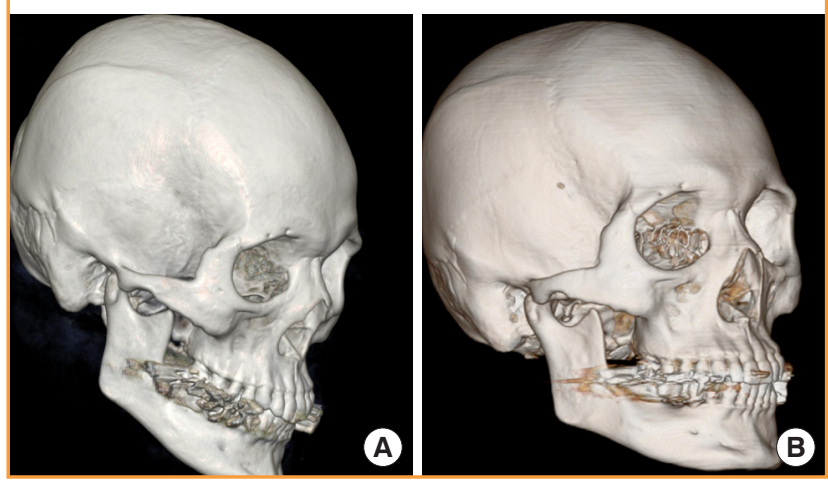

\section{RESULTS}

The postoperative CT images showed that the titanium mesh was well-inserted and in the proper position, and that the orbital contents were kept in the orbit. We also confirmed that the symptoms that patients complained of before surgery had resolved after surgery. All patients were discharged within 1 week after surgery without any complications.

\section{DISCUSSION}

In 1991, Yamaguchi et al. [6] introduced the endoscopic transnasal approach for reconstruction of medial orbital wall fractures; since then, it has been widely used due to its various advantages, including a wide visual field during the operation and the lack of a subsequent scar on the facial area [7-11]. However, the choice of the implant to be inserted is also important, as well as the approach to the reduction of medial orbital wall fractures. The implant should be determined considering the defect size, associated injuries, age, and donor site morbidity [17]. The ide- 
al orbital implant is biocompatible, easy to obtain, strong enough to provide orbital support, easy to handle, and radiopaque [18]. Various materials have been introduced for the treatment of orbital wall fractures. They can be broadly divided into autologous and alloplastic materials. Alloplastic materials are widely used because they have no donor site morbidity, can reduce the operating time, and are easy to use $[19,20]$.

The combination of a silastic sheet and Merocel used with the endoscopic transnasal approach for reconstruction of the medial orbital wall is cheap, easy to handle, and can provide adequate support for large defects. However, techniques that use these materials require them to be kept in place for a long time, which makes it impossible to avoid patient discomfort and may cause surgical site infection [21]. In the meantime, a method of positioning an absorbable plate in orbit through the transnasal approach has been introduced [12]. Absorbable plates are easy to handle due to their malleability and have the advantage of not requiring removal. However, they may lose their load-bearing capacity before complete healing, and it is difficult to confirm the location of absorbable plates by imaging because of their radiolucency $[13,14]$.

Because of these problems, the authors devised a new surgical technique for inserting a radiopaque nonabsorbable plate into the orbit by the endoscopic transnasal approach. A nonabsorbable plate is harder and difficult to handle than an absorbable plate, so it was trimmed to be shorter than the anteroposterior length of the defect and longer than its height so that the implant could be inserted through the bone defect site. It could be possible to trim the plate to be shorter than the height and longer than the anteroposterior length of the defect. However, when the plate is inserted, the tissue around the bone defect could be damaged by the plate, and we were afraid of damage to the optic nerve. Therefore, the authors chose the former strategy. This allowed the implant to be fixed in proper position by the intact bone around the defect. This method does not require nasal packing, and therefore reduces the risk of complications such as infection and patient discomfort. Additionally, titanium mesh with porous polyethylene has the advantage of being able to maintain its load-bearing capacity permanently, and its position can be identified on postoperative radiological examinations. However, the limitations of this technique are that it is difficult to perform, the operating time is relatively long, and it is likely to be influenced by the skill of the operator. It may also be expected that the mesh will be difficult to remove after insertion. In this study, we confirmed that excellent results were obtained from CT examinations performed immediately after surgery. However, a disadvantage is that no follow-up for more than 6 months was carried out, and no study has explored sub- sequent complications. The small number of cases is also a limitation of this study. Further research on these issues will be needed in the future, but this surgical technique is expected to be able to overcome the problems of other surgical techniques.

The authors have developed a surgical method of inserting a nonabsorbable titanium mesh with porous polyethylene into the orbit through a transnasal endoscopic approach, and obtained good results, as confirmed by postoperative CT scans. Although additional study is needed, this technique is expected to be another surgical option for medial orbital wall fractures.

\section{NOTES}

\section{Conflict of interest}

No potential conflict of interest relevant to this article was reported.

\section{Ethical approval}

This study was approved by the Institutional Review Board of Pusan National University Hospital (IRB No. H-1907-003080) and was performed in accordance with the principles of the Declaration of Helsinki. Written informed consent was obtained.

\section{Patient consent}

The patients provided written informed consent for the publication and the use of their images.

\section{Author contribution}

Conceptualization: Bae SH, Jeong DK. Data curation: Go JY, Park H. Formal analysis: Kim JH. Methodology: Lee JW. Project administration: Kang T. Writing - original draft: Jeong DK. Writing - review \& editing: Bae SH. Approval of final manuscript: all authors.

\section{ORCID}

Seong Hwan Bae https://orcid.org/0000-0002-7203-8978

Dae Kyun Jeong https://orcid.org/0000-0002-6379-2629

Ju Young Go https://orcid.org/0000-0002-5808-1806

Heeseung Park https://orcid.org/0000-0001-9889-5217

Joo Hyoung Kim https://orcid.org/0000-0002-4893-3761

Jae Woo Lee https://orcid.org/0000-0002-0945-6966

Taewoo Kang https://orcid.org/0000-0002-6279-0904

\section{REFERENCES}

1. de Visscher JG, van der Wal KG. Medial orbital wall fracture with enophthalmos. J Craniomaxillofac Surg 1988;16:55-9. 
2. Hwang K, You SH. Analysis of facial bone fractures: an 11year study of 2,094 patients. Indian J Plast Surg 2010;43:428.

3. Nolasco FP, Mathog RH. Medial orbital wall fractures: classification and clinical profile. Otolaryngol Head Neck Surg 1995; 112:549-56.

4. Lee CS, Yoon JS, Lee SY. Combined transconjunctival and transcaruncular approach for repair of large medial orbital wall fractures. Arch Ophthalmol 2009;127:291-6.

5. Lee WT, Kim HK, Chung SM. Relationship between smallsize medial orbital wall fracture and late enophthalmos. J Craniofac Surg 2009;20:75-80.

6. Yamaguchi N, Arai S, Mitani H et al. Endoscopic endonasal technique of the blowout fracture of the medial orbital wall. Oper Tech Otolayngol Head Neck Surg 1991;2:269-74.

7. Jeon SY, Kim C, Ma Y, et al. Microsurgical intranasal reconstruction of isolated blowout fractures of the medial orbital wall. Laryngoscope 1996;106:910-3.

8. Sanno T, Tahara S, Nomura T, et al. Endoscopic endonasal reduction for blowout fracture of the medial orbital wall. Plast Reconstr Surg 2003;112:1228-37.

9. Lee MJ, Kang YS, Yang JY, et al. Endoscopic transnasal approach for the treatment of medial orbital blow-out fracture: a technique for controlling the fractured wall with a balloon catheter and Merocel. Plast Reconstr Surg 2002;110:417-26.

10. Rhee JS, Chen CT. Endoscopic approach to medial orbital wall fractures. Facial Plast Surg Clin North Am 2006;14:1723.

11. Jin HR, Yeon JY, Shin SO, et al. Endoscopic versus external repair of orbital blowout fractures. Otolaryngol Head Neck Surg 2007;136:38-44.

12. We J, Kim Y, Jung T, et al. Modified technique for endoscopic endonasal reduction of medial orbital wall fracture using a resorbable panel. Ophthalmic Plast Reconstr Surg 2009;25: 303-5.

13. Han DH, Chi M. Comparison of the outcomes of blowout fracture repair according to the orbital implant. J Craniofac Surg 2011;22:1422-5.

14. Losken HW, van Aalst JA, Mooney MP, et al. Biodegradation of Inion fast-absorbing biodegradable plates and screws. J Craniofac Surg 2008; 19:748-56.

15. Al-Sukhun J, Tornwall J, Lindqvist C, et al. Bioresorbable poly-L/DL-lactide (P[L/DL $] \mathrm{LA} 70 / 30)$ plates are reliable for repairing large inferior orbital wall bony defects: a pilot study. J Oral Maxillofac Surg 2006;64:47-55.

16. Baek WI, Kim HK, Kim WS, et al. Comparison of absorbable mesh plate versus titanium-dynamic mesh plate in reconstruction of blow-out fracture: an analysis of long-term outcomes. Arch Plast Surg 2014;41:355-61.

17. Belli E, Matteini C, Mazzone N. Evolution in diagnosis and repairing of orbital medial wall fractures. J Craniofac Surg 2009;20:191-3.

18. Dubois L, Steenen SA, Gooris PJ, et al. Controversies in orbital reconstruction-III: biomaterials for orbital reconstruction: a review with clinical recommendations. Int J Oral Maxillofac Surg 2016;45:41-50.

19. Schubert W, Gear AJ, Lee C, et al. Incorporation of titanium mesh in orbital and midface reconstruction. Plast Reconstr Surg 2002;110:1022-30.

20. Reich W, Seidel D, Bredehorn-Mayr T, et al. Reconstruction of isolated orbital floor fractures with a prefabricated titanium mesh. Klin Monbl Augenheilkd 2014;231:246-55.

21. Kim YH, Park Y, Chung KJ. Considerations for the management of medial orbital wall blowout fracture. Arch Plast Surg 2016;43:229-36. 